Three-dimensional modeling and analysis of a high energy density Kelvin-Helmholtz experiment

K. S. Raman, O. A. Hurricane, H. S. Park, B. A. Remington, H. Robey, V. A. Smalyuk

February 22, 2012

Physics of Plasmas 
This document was prepared as an account of work sponsored by an agency of the United States government. Neither the United States government nor Lawrence Livermore National Security, LLC, nor any of their employees makes any warranty, expressed or implied, or assumes any legal liability or responsibility for the accuracy, completeness, or usefulness of any information, apparatus, product, or process disclosed, or represents that its use would not infringe privately owned rights. Reference herein to any specific commercial product, process, or service by trade name, trademark, manufacturer, or otherwise does not necessarily constitute or imply its endorsement, recommendation, or favoring by the United States government or Lawrence Livermore National Security, LLC. The views and opinions of authors expressed herein do not necessarily state or reflect those of the United States government or Lawrence Livermore National Security, LLC, and shall not be used for advertising or product endorsement purposes. 


\title{
Three-dimensional modeling and analysis of a high energy density Kelvin-Helmholtz experiment.
}

\author{
K. S. Raman, O. A. Hurricane, H.-S. Park, B. A. Remington, H. Robey, and V. A. Smalyuk \\ Lawrence Livermore National Laboratory, Livermore, CA 94550 \\ R. P. Drake, C. M. Krauland, C. C. Kuranz \\ University of Michigan, Ann Arbor, MI 48109 \\ J. F. Hansen \\ General Atomics, San Diego, CA 92121 \\ E. C. Harding \\ Sandia National Laboratory, Albuquerque, NM 87125
}

(Dated: August 27, 2012)

\begin{abstract}
A recent series of experiments on the OMEGA laser provided the first controlled demonstration of the KelvinHelmholtz instability in a high-energy-density physics context (E. C. Harding, et al., Phys. Rev. Lett., 103, 045005,2009; O. A. Hurricane, et al., Phys. Plasmas, 16, 056305, 2009). We present 3D simulations which resolve previously reported discrepancies between those experiments and the 2D simulation used to design them. Our new simulations reveal a three-dimensional mechanism behind the low density "bubble" structures which appeared in the experimental $\mathrm{x}$-ray radiographs at late times but were completely absent in the 2D simulations. We also demonstrate that the three-dimensional expansion of the walls of the target is sufficient to explain the $20 \%$ overprediction by $2 \mathrm{D}$ simulation of the late-time growth of the $\mathrm{KH}$ rollups. The implications of these results for the design of future experiments is discussed.
\end{abstract}

\section{INTRODUCTION}

We present a three-dimensional simulation of a novel experimental platform, which enabled the first controlled observation and analysis of the Kelvin-Helmholtz (KH) instability in a high-energy-density (HED) physics context[1-4]. The KH experiments were notable for their reproducibility and high quality data, so there is considerable momentum to utilize this platform to investigate other aspects of HED shear flows. A program is currently underway to see how such experiments may be used to calibrate turbulent mix models in the HED regime $[5,6]$. The present work is part of a parallel effort to refine our model of the basic platform to inform the design of future experiments.

The basic idea of the Kelvin-Helmholtz experiments is summarized in Fig. 1 and the target geometry and configuration are shown in Fig. 2. A detailed discussion of the reasoning behind various facets of the design is given in Ref. 1 . As shown in Fig. 1, the platform involves launching a laserdriven shock into a low-density $(0.1 \mathrm{~g} / \mathrm{cc})$ carbon foam, along a pre-modulated interface with a high-density plastic (1.42 $\mathrm{g} / \mathrm{cc}$ ). The shock converts the foam to a plasma and gives it a shear velocity relative to the plastic, as the plastic itself is also turned into a plasma by the transmitted shock accompanying the main shock. As the shock moves through the foam, a large vortex sheet is created at the modulated interface, which now separates two fluids. The latter may be seen by examining the vorticity equation for an inviscid, compressible fluid[7]:

$$
\frac{D \omega}{D t}=\frac{\nabla \rho \times \nabla P}{\rho^{2}}
$$

The symbols have their usual meanings, i. e. $t$ is time, $\omega$ the vorticity, $\frac{D}{D t}$ the convective derivative, $\rho$ the density, and $P$ the pressure. The source term on the right-hand-side, commonly called the baroclinic torque, would be especially large at the shock front near the fluid interface, due to the near orthogonality of the large pressure gradient across the shock with the large density gradient at the interface. Simulation confirms that most of the vorticity generated within the time scale of the experiment is, in fact, created at the interface during shock passage via this baroclinic mechanism.

As the vortex sheet develops in the plasma shear flow into the characteristic $\mathrm{KH}$ rollup structure, backlighter $\mathrm{x}$-rays radiograph the process from the side, as illustrated in Fig. 2. The materials are contained in a beryllium shock tube of rectangular cross section, and an opaque, density-matched, tracer strip in the plastic ensures the visualization of a 2D slice of the 3D flow. Fig. 3a is an example of an experimental radiograph which was obtained by this approach.[2, 3].

The timing, size, and shape of the KH vortices seen in the experiments were in basic agreement with the 2D design simulation (see Fig. 3b and Refs. 2-4), but there were some discrepancies, as well as features seen in the experiments but not in the simulation. Some of these were fine features, such as the breakup of the arms of the KH rollups at late times (compare Figs. $3 \mathrm{a}$ and $3 \mathrm{~b}$ ), that are likely due to microscopic processes not being modeled, such as turbulent mixing. However, there were two noteworthy "large-scale" discrepancies, which our 3D simulation has now enabled us to explain.

First, and most striking, were growing low density "bubble" structures, which appeared in the experimental x-ray images at late times above the largest vortices, as shown in Fig. 3a. A number of speculations were offered[3] for what these structures might be including evidence of a phase transition not captured by the model equation of state; localized "shocklets" related to the transonic Mach numbers involved[8]; and cavitation-like features, the origins of which were unclear. The second issue was an overprediction, by about $20 \%$, of 


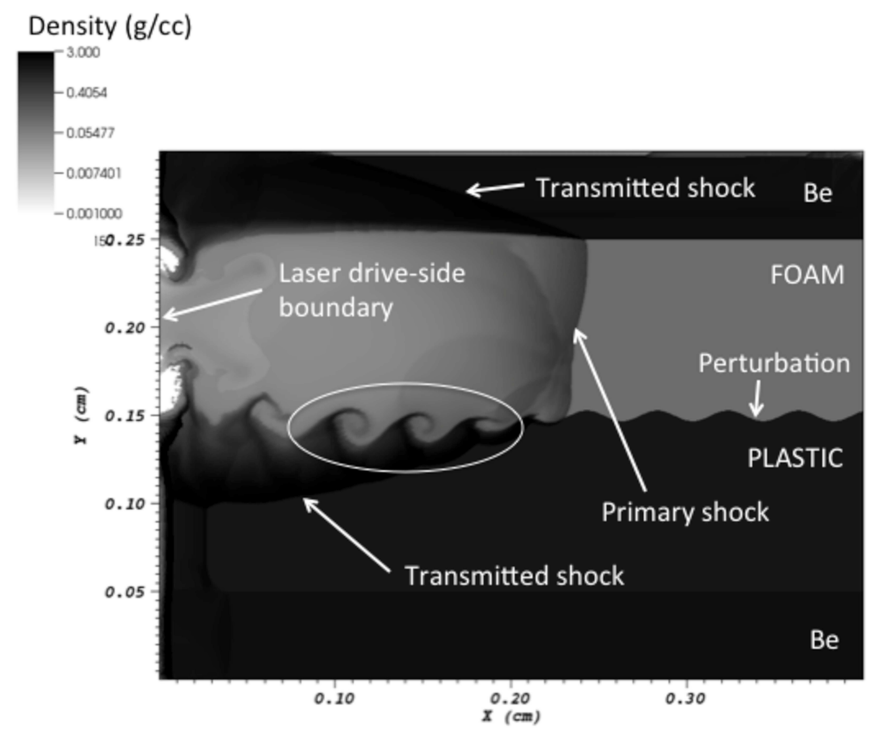

FIG. 1: Density contour plot from a 2D ARES simulation at around $40 \mathrm{~ns}$, where the density is plotted on a logarithmic color scale for visualization. The experimental field of view is indicated by the circle. The simulated flow was initiated by an energy source depositing $4 \mathrm{~kJ}$ in $1 \mathrm{~ns}$ on the laser drive-side boundary (indicated). KH vortices at varying stages of evolution are evident. As the primary shock moves in the low density foam, transmitted shocks are seen moving in the high density plastic and the beryllium wall of the shock tube. The upper left corner shows the transmitted shock in the beryllium beginning to blow through the top wall. The leading edge where the shock front meets the pre-perturbed interface is where the most of the vorticity in this experiment is generated (see Eq. 1).

the late-time vortex growth by the simulation. It was noted[4] that three-dimensionality could be relevant here, as the postshock expansion of the side walls of the shock tube, which a 2D simulation would not capture, could cause a sideways stretching of the vortex rollups which, by mass conservation, would reduce the extent of vertical growth.

The main conclusions of this work are summarized in Figs. 3 and 4. Fig. 3 compares a late-time ( $75 \mathrm{~ns}$ ) experimental x-ray radiograph (3a) with synthetic x-ray images obtained from 2D (3b) and 3D (3c) simulations. Low density regions appear above the crests at late-times in the 3D simulation, as in the experiment, but not in the 2D simulation. Fig. 4 plots, for each of the three crests visible in the experimental field of view, the peak-to-valley height versus time elapsed after the crest was hit by the shock. While both the 2D and 3D simulation agree well with experiment at early times, at late times, the 3D simulation predicts a noticeably lower growth rate, consistent with experiment.

The rest of this paper is devoted to presenting the physical mechanisms which underly our improved agreement with experiment. In Section II, we overview the models and simulations used. Section III presents the mechanism behind the low-density bubbles and section IV discusses the growth of the KH rollups and why 3D simulations predict lower growth rates. These two sections show that the outstanding physics issues of the previously reported discrepancies are largely re-

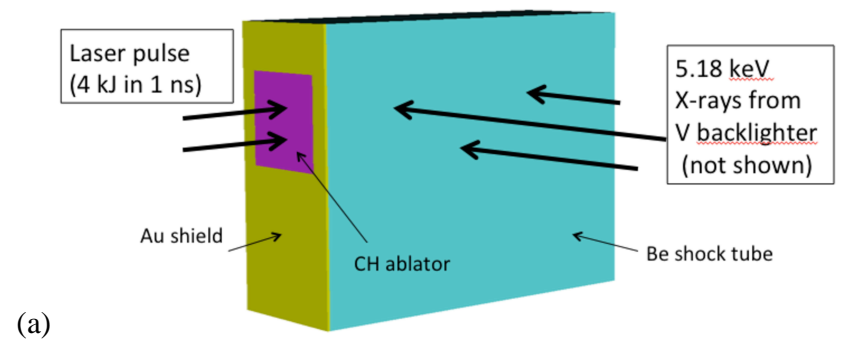

(a)

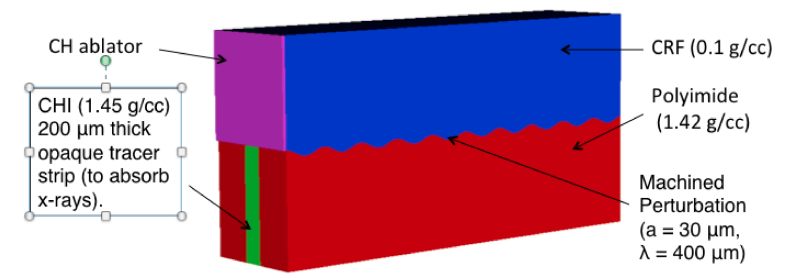

(b)

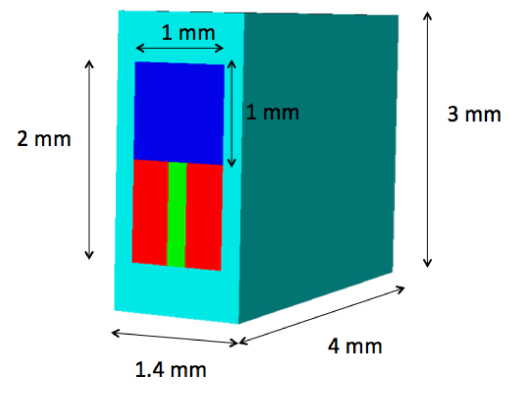

(c)

FIG. 2: Views of the target configuration used in the experiments. (a) The experiment occurs inside of a beryllium shock tube of rectangular cross-section. A square laser pulse delivers $4 \mathrm{~kJ}$ in $1 \mathrm{~ns}$ to a 30 $\mu \mathrm{m} \mathrm{CH}$ ablator covering the foam section while the rest of the target is protected by a thin gold shield. Backlighter $\mathrm{x}$-rays radiograph the target from the side. (b) The gold shield and beryllium walls are now removed revealing the perturbed plastic-foam interface inside, as well as the $200 \mu \mathrm{m}$ thick opaque radiographic tracer layer. (c) This is a view inside the beryllium shock tube, where the walls are shown, but the gold shield and $\mathrm{CH}$ ablator have been removed. Various dimensions of interest have been labeled.

solved. The work of using these insights to refine the target design and to develop higher fidelity models of the integrated experiment is still in progress. However, some implications of our results for such efforts is discussed in section V.

\section{MODEL AND SIMULATIONS}

The 2D simulations presented here were performed using the C-based Arbitrary Lagrangian-Eulerian (CALE) code[9] or the Advanced Strategic Computing code ARES[10], both of which were developed at LLNL. The 3D simulation, performed using ARES, involved nearly 13 million zones, and ran for $130+$ hours with 4128 processors, using the supercomputing facilities at LLNL. This corresponds to a spatial resolution in the target of roughly $7 \mu \mathrm{m} \times 15 \mu \mathrm{m} \times 20 \mu \mathrm{m}$ (length $\mathrm{x}$ height $x$ thickness). Computer resource limitations prevented 
us from exploring finer resolutions. According to our 2D simulations, this $x$ and $y$ resolution is sufficient to resolve the overall shape of the vortical structures, though a resolution of at least $4.5 \mu \mathrm{m} \times 10 \mu \mathrm{m}$ (length $\times$ height) is needed to begin to resolve the structure inside a rollup. The $3 \mathrm{D}$ simulation was post-processed using the HADES code[11] to obtain a synthetic $\mathrm{x}$-ray radiograph (Fig. 3c), accounting for camera angles and diagnostic resolution.

Both of these codes use arbitrary Lagrangian-Eulerian (ALE) methods to handle the hydrodynamic mesh motion and advection. The simulations included single-group radiation diffusion, electron heat conduction, tabulated equations of state and opacities, and ionization based on a ThomasFermi model. The CALE simulation modeled the laser by depositing energy at a critical surface determined by the electron plasma frequency. As reported previously[3], the shock timing predicted by this approach closely matched experiment. The ARES simulation modeled the laser indirectly by applying an energy source, with magnitude and time profile roughly based on the CALE results, to the outer zones of the ablator.

In addition to the simulation, our data interpretation is based on viewing the disturbance created by the laser in the foam, composed of a shock followed by a rarefaction, as a 1D blast wave. The model appears twice in our analysis. First, the energy source of the 3D simulation presented here actually corresponds to a laser deposition of $8.5 \mathrm{~kJ}$ in $1 \mathrm{~ns}$, compared to the $4 \mathrm{~kJ}, 1 \mathrm{~ns}$ pulse used in the experiment (computer resource limitations prevented recalculation with $4 \mathrm{~kJ}$ ). Therefore, when we compare our 3D results with experiment or other simulations at different energies (as in Figs. 3 and 4), we scale the time by a factor $E^{1 / 2}$, as suggested by the selfsimilar motion of a 1D blast wave. Second, we are interested in how the heights of the KH structures evolve after the shock has passed. Experimental radiographs were obtained at $25 \mathrm{~ns}$, $45 \mathrm{~ns}$, and $75 \mathrm{~ns}[2,3]$ but the shock only appeared in the $25 \mathrm{~ns}$ frame. Therefore, the blast wave model of the shock motion, $\mathrm{i}$. e. that the shock position $X_{s}$ is proportional to $(E / \rho)^{1 / 3} t^{2 / 3}$ where $E$ is the energy deposited per unit area, $\rho$ the foam density, and $t$ the time, calibrated to the $25 \mathrm{~ns}$ frame, permits us to infer when the shock first passed a given crest.

It is difficult to quantify the uncertainty introduced by interpreting the data through the blast wave picture. Experimental resource limitations, including the fact that typically only one radiograph is obtained per experiment, prevented a purely experimental assessment of both the shock dynamics and vortex growth. Also, as mentioned, computer resource limitations prevented a thorough exploration of the 3D parameter space. We have verified the validity of the $1 \mathrm{D}$ blast wave approximation for the shock motion in both the $2 \mathrm{D}$ and $3 \mathrm{D}$ simulation on the time scales of the experiment. The similarity of radiographs taken at different times with different foam densities in Ref. 4 provides additional experimental support for the blast wave picture. However, given these caveats, we place greater emphasis on the physical mechanisms uncovered and their orders of magnitude, than the literal agreement of our simulated data with experiment (Fig. 4).

\section{THE ORIGIN OF THE BUBBLES}

A useful starting point for understanding the bubbles is to examine a lengthwise slice of the target, as shown in Fig. 5. Fig. 5a shows the beryllium wall and modulated plastic section of the undisturbed target; the foam region, which sits above the plastic, has been removed for visualization. As time advances, the laser-driven shock propagates from left to right in the (removed) foam sequentially perturbing the plastic crests, which develop into the characteristic KH rollups. Shadows of this shock are apparent in these images as the transmitted shocks moving in the plastic and beryllium regions.

It is important to note from Fig. 5a that initially only the plastic region is modulated while the beryllium inner side wall is a flat surface. However, as the shock propagates over each crest, it appears to be punching holes in the beryllium wall, effectively imprinting the modulation onto it. What is happening here can be understood in a number of ways. One way is to note that as the shocked fluid flows "up" each crest of the modulation, its vertical extent decreases causing it (by its essential incompressibility due to the fairly low post-shock Mach number) to exert a sideways pressure on the beryllium walls, and vice versa as it flows "down" the other side. Another way is to note that the shock does not literally move at right angles to the foam-plastic interface but is an oblique shock at the interface due to the nonzero amplitude of the perturbation. While this does not influence the freestream shock motion, the pressure jump across the shock will be larger as the shock climbs and smaller as it falls as its oblique shock angle varies over the crest.

The effect is not small and, in retrospect, can be seen indirectly in the 2D simulation. Fig. 6 shows pressure contour plots from a 2D ARES simulation focused on the region near the foam-plastic interface as the shock climbs over one of the crests. The pressure variation, indicated by the arrows, is not small and may be greater than even the freestream pressure. For instance, moving forward in time from Fig. $6 \mathrm{a}$ to $6 \mathrm{~b}$ to $6 \mathrm{c}$, the freestream pressure jump across the shock drops from $\sim 1.2$ Mbar to $\sim 1.0$ Mbar to $\sim 0.9$ Mbar, consistent with blast wave decay. However, near the interface, the pressure jump is 23.5 Mbar during the first climb (a), falls to $~ 0.44$ Mbar during the first fall (b), and then rises to $\sim 2.4$ Mbar during the second climb (c). This large additional pressure modulation is what imprints the plastic perturbation onto the Be side walls lying above and below the page.

Another perspective on this imprinting is gained from Fig. 7. Here, we have shown one of the frames from the high-resolution 2D CALE simulation discussed in Ref. 1. As the primary shock passes over each crest, spherical reflected shocks are emitted due to the aforementioned shock angle at the interface not being exactly 90 degrees. These reflected waves are also transmitted into the beryllium side walls (as well as the plastic) in addition to the transmitted shocks associated with the primary wave.

We now show the relevance of this to the "bubbles". Fig. 8a shows the undisturbed target from the same point of view as Fig. 5a, except now all materials have been removed except 
the beryllium wall. The front wall of the shock tube has been cut to the height of the foam-plastic interface.

Focusing on the back wall, the time sequence shows, as before, a shadow of the shock as it moves down the channel, writing the plastic modulation onto the beryllium. Focusing on the front wall, Fig. $8 \mathrm{~b}$ is an early time snapshot showing a small amplitude perturbation imprinted onto the wall in the field-of-view of the experiment. At this time, the transmitted shock is just about to blow through the thin side wall. By Fig. 8c, the transmitted shock has blown through the thin side walls and is about to blow through the thicker top wall. Once the side wall has been blown through, the imprinted perturbation becomes exposed to the massive pressure and velocity gradients between the high-speed, high-pressure fluid inside the channel and outside vacuum. The instability growth of this perturbed foam-beryllium interface leads to low density foam bubbles poking through the thin beryllium wall, as apparent in Figs. 8d-f.

Figs. 9 and 10 give different perspectives on this effect. Fig. 9 is a late-time image of just the foam and plastic parts, visually confirming the sideways growth of the foam. Fig. 10 is a side view of the beryllium shock tube at late time, where only zones with density greater than $1 \mathrm{~g} / \mathrm{cc}$ have been drawn. In this latter type of plot, locations where the foam bubbles have broken through appear as "holes" in the shock tube. Backlighter x-rays passing through one of these "holes", actually low density foam regions, experience less optical depth than $\mathrm{x}$-rays passing through denser beryllium sections. This modulation in optical depth, corresponding to the modulated blowout of foam bubbles through the beryllium wall, is the basic cause for the low density structures appearing in the experimental and simulated radiographs (Fig. 3a and c). In conclusion, the "bubbles" in the radiograph are seen to be literally bubbles of foam blowing through holes in the shock tube.

\section{THE OVERPREDICTION OF VORTEX GROWTH}

The reason why the vortex growth rates predicted by $3 \mathrm{D}$ simulation are substantially lower than for 2D simulation (see Fig. 4) is explained in Fig. 11. Fig. 11 shows filled material boundary plots of the plastic section, with the opaque tracer strip colored differently. Fig. 11a is an early time slice showing structures just beginning to develop. In this frame, the height of the forming rollup is greater at the center than the edges. By Fig. 11b, the center of the rollup has started to become slightly depressed compared to the sides and by the time shown in Fig. 11c, the depression is substantial.

As the primary shock propagates down the channel, transmitted shocks propagate into the plastic and beryllium walls, while reflected shocks are launched back into the foam. It was previously noted[4] that the impact of the reflected shock from the top beryllium wall on the foam-plastic interface correlated with a noticeable reduction in the vortex growth rate seen in 2D simulation. We observe that the centerline depression begins near the time the two reflected shocks from the beryllium side walls cross one another. Prior to this time (i.e. Fig. 11a), the fluid above the centerline would be at a lower pressure than the side fluid, which had experienced the reflected shock. However, when the reflected shocks cross one another, the twice-shocked central foam would be at higher pressure than the once-shocked side foam.

This outward lateral pressure gradient persists till late time due to the side wall expansion. Hence, the centerline depression grows in time, as seen in Fig. 11c. If the side walls were rigid, we would expect the lateral pressure gradient to heal itself as the side fluid experiences its second reflected shock, and perhaps reverse itself as that shock is reflected again. However, the side wall expansion, which begins soon after the reflected shock, rapidly brings the pressure at the sides to essentially vacuum and the material is pulled outwards. When the two side wall rarefactions cross the center of the channel, there is significant sideways expansion of the central fluid which, by mass conservation, leads to a corresponding reduction in vertical growth.

These images demonstrate that the dynamics of the side wall is a quantitatively significant effect not captured in a 2D simulation. They show that the center of the target gets especially impacted at late times, consistent with the results of Fig. 4. Positioning the tracer strip off the centerline might be an interesting experiment to provide additional verification of this mechanism.

\section{DISCUSSION}

The previous sections have shown that both the "bubbles" and the vortex height overprediction arise from the interaction between the shocked fluid and the walls of the shock tube. The mechanisms discussed above also suggest possible ways to eliminate these edge effects, which would improve the precision of this platform as a tool for validating two-dimensional simulations.

The bubbles can be mitigated by a reduction in the instability growth and/or the imprinting process. The imprinting process can be weakened by reducing the amplitude of the pre-machined plastic perturbation, which would make the shock angles at the interface closer to perpendicular. According to the analysis of Ref. 1, the nonlinear vortex growth is not sensitive to the initial amplitude so this sort of change would not compromise other advantages of the design, provided the amplitude is large enough that the nonlinear phase is entered quickly. The instability growth is especially rapid after the shock blows through the thin wall, exposing the foamberyllium interface to very large pressure and velocity gradients. A natural way to delay this would be to thicken the walls or otherwise increase their rigidity by changing the material, though both of these changes would affect the transparency of the target walls to x-rays. The tradeoff between having thicker walls and achieving sufficient signal-to-noise for the $\mathrm{x}$-ray radiography needs to be explored. However, making the side walls more rigid could also impact the reflected shock dynamics, as noted in Section IV.

Another possibility would be to use a wider target to further insulate the centerline from the side walls. However, a convenient feature of the existing design is that the propa- 
gating shock is a $1 \mathrm{D}$ blast wave, which simplifies the data interpretation. To achieve a 1D blast wave with a substantially wider target would require significantly more laser energy that, nonetheless, might be attainable on NIF.

While our focus has been on the side walls, another source of edge effects is the laser drive-side boundary. The experimental field-of-view was focused 1-2 mm downstream of this boundary to avoid the messy flow field near the boundary, suggested by simulation[1]. Continuing this analysis, Fig. 12 shows late-time density plots from 2D simulations using (a) CALE and (b) ARES, the only differences between them being the model for laser energy deposition and aspects of the ALE strategy used to control the mesh during the ablation phase. While these choices are not important for broad features such as the shock motion or overall vortex growth, it does influence the nuances of the flow field near the driveside boundary. An observable consequence of this is that the crests in the 1-2 mm field-of-view have a larger height-towidth aspect ratio in (a) and than in (b), but become more similar in shape further downstream. Our aim is not to decide which picture is more accurate, which involves issues of numerical resolution as well as physics (indeed, while (a) models the laser energy deposition with greater fidelity, the shapes of the rollups in (b) more closely resembles experiment; neither model fully resolves the ablation physics). The purpose was to demonstrate the subtle ways in which boundary conditions can influence the results.

Finally, we comment on the vortex growth rate. If the shock moved with constant velocity, as in a conventional pressuredriven shock tube, the growths of the different crests would be identical and related by simple time shifts corresponding to the shock passage interval. However, the shock is actually a decaying wave so relating the growths of different crests to one another requires additional scaling. The shock motion itself is well-modeled as a conventional 1D blast wave, $X_{\mathrm{S}} \sim$ $\left(\frac{E}{\rho}\right)^{1 / 3} t^{2 / 3}$, on the time scales of the experiment, though at late times, a smaller exponent than $2 / 3$ might be more accurate (for instance, 3/5 was used in Ref. 2), as the flow more closely resembles an impulsively-driven problem[13]. Here $X_{s}$ is the shock position, $t$ the time, $E$ is the energy deposited per area and $\rho$ the foam density.

We may postulate a scaling form for the vortex growth by analogy with the $1 \mathrm{D}$ blast wave. Assume the relevant variables affecting the peak-to-valley height $h$ of a rollup are its downstream position $x$, the time $t$ after the crest was hit by the shock, the energy per unit area $E$, and a density scale $\rho$ related to the foam and plastic densities. We can form a nondimensional variable from these quantities, $\eta \equiv\left(\frac{E}{\rho}\right)^{1 / 3} \frac{t^{2 / 3}}{x}$. The height $h$ of a crest will scale as $x$ times a function of this variable. The results in Fig. 4 are plotted in this manner in Fig. 13. The 2D data almost collapse on the same curve, while the 3D data, which agree better with experiment, do not.

The 2D data collapse is not perfect, the deviation likely due to real features being modeled. For example, the above picture presumes the rollups grow independently of one another but when the size of a rollup becomes comparable to the spacing between rollups, i. e. the wavelength of the imposed perturbation $\lambda$, we might expect the presence of neighboring rollups to have an impact on the growth. The 2D data roughly fall on the same curve suggesting the idealized picture is a decent approximation on the time scales we are concerned with. However, at even later times, when the rollups are large, the flow may be better described via a strong coupling model such as the line vortex approach of Refs. 1, 14.

The roughly linear trend of the 2D data after early times shows that $h \sim t^{2 / 3}$. This power can be understood by noting that the shear velocity above a crest will approximately scale as $t^{-1 / 3}$, the same as the shock velocity, at late enough times[16]. Therefore, the $2 / 3$ power can be understood as $h$ being proportional to the time integral of the velocity shear, which is what one would expect for a Kelvin-Helmholtz process. If the dominant process were more RichtmeyerMeshkov like, we would expect $h \sim t$, as the growth would be primarily induced by the velocity impulse delivered by the shock and, due to the near unity Atwood number, the plastic "spike" would fall at constant velocity[15].

Unlike the 2D data, the 3D data do not collapse when plotted in these coordinates. In 3D, we have an additional relevant length scale, the side wall thickness, which does not appear in 2D. We might expect self-similarity to return if this side wall were replaced by a free boundary, which is a topic of future study[17].

\section{CONCLUSION}

In conclusion, we have presented our three-dimensional simulation of a novel Kelvin-Helmholtz experiment which resolved some puzzling features from two previous experimental campaigns. It was shown that all of the outstanding macroscopic features are, in fact, explained by physical processes already modeled by our codes, when the modeling is done in 3D. This provides a crucial validation of our basic approach as we move forward with this platform to explore uncharted aspects of HED shear flows[5, 6].

\section{Acknowledgments}

We would like to acknowledge B. Pudliner and R. Tipton for valuable technical input during the development of our 2D and 3D ALE strategies. We acknowledge M. J. Bono, R. Gillespie, and M. Grosskopf, for important contributions to the experimental campaigns mentioned in the text. We thank the following individuals for useful discussions during the course of this study: P. Amala, G. Archbold, T. S. Carman, R. Cook, D. Cotrell, Y. Elbaz, J. Greenough, D. Laney, A. R. Miles, P. L. Miller, J. C. Moreno, T. Orzechowski, and D. Shvarts. This work was performed under the auspices of the U.S. Department of Energy by Lawrence Livermore National Laboratory under Contract DE-AC52-07NA27344. Work at Michigan is supported by the NNSA-DS and SC-OFES Joint Program in High-Energy-Density Laboratory Plasmas, grant number DE-FG52-09NA29548, and by the National Laser User Facility Program, grant number DE-FG52-09NA29034. 


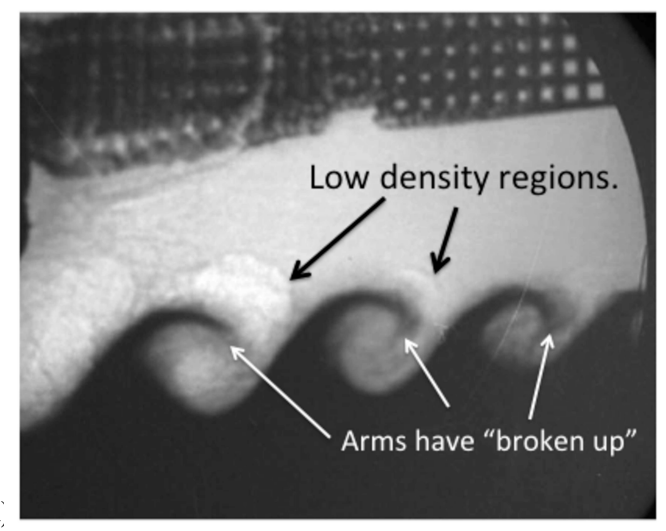

(a

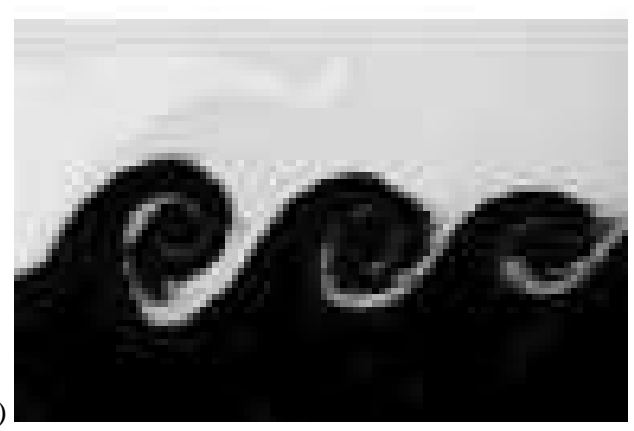

(b)

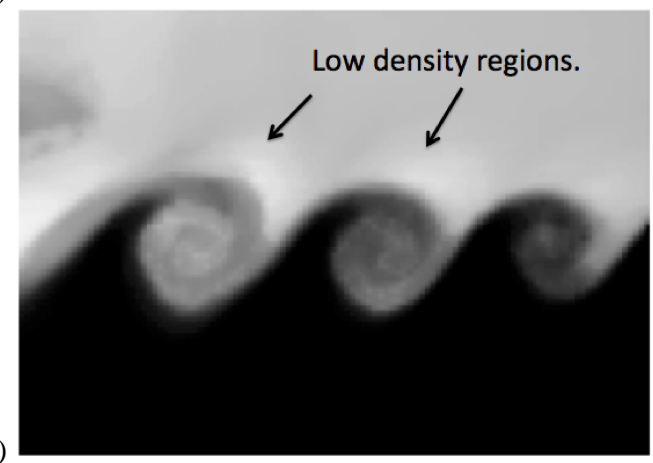

FIG. 3: Comparison of the late-time (75 ns) $\mathrm{x}$-ray radiograph from the (a) May 2008 experiment[2, 3], with synthetic $x$-ray images from (b) 2D CALE and (c) 3D ARES simulations. The field-of-view, circled in Fig. 1, is approximately 1-2 $\mathrm{mm}$ downstream of the laser drive-side boundary. Note the low density regions which appear above the crests in the experiment (a) and 3D simulation (c), but not in the 2D simulation (b). The explanation of these bubble-like structures is one of the key results of this work. The arms of the experimental rollups appear to break up (a), while the simulated rollups are fuller (b and c). This is likely due to microscopic processes, such as turbulent mixing, which were not included in the simulation. The synthetic radiographs are plotted in a linear gray scale, the $\mathrm{x}-$ ray absorption in the foam being about $30 \%$ of the absorption in the plastic, and approximately $15 \%$ in the low density regions. We did not attempt to model the diagnostic, beyond accounting for the 17 $\mu$ m pinhole blurring, to make a quantitative comparison with the experimental radiograph. On one hand, our simulation was relatively coarse in the out-of-plane dimension, which, as the present work shows, plays the crucial role in creating the low density regions. On the other hand, Ref. 4 showed that the degree of contrast with which these low density regions appear in the experimental radiographs varied from shot to shot. The analysis in Ref. 6 suggests this variability might be due to sensitivity of these features to the surface roughness of the beryllium walls, which was not included in our simulation. The radiograph in Fig. 3a is reprinted with permission from Phys. of Plasmas 16, 056305 (2009); Copyright 2009 American Institute of Physics. 


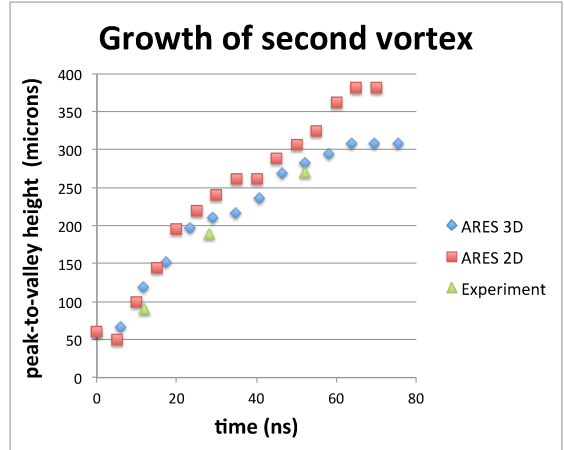

(a)

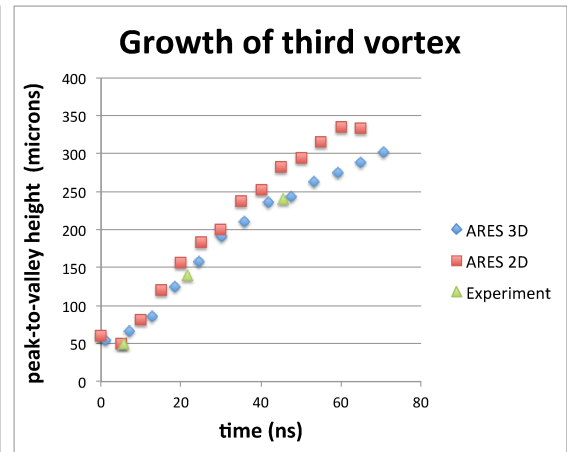

(b)

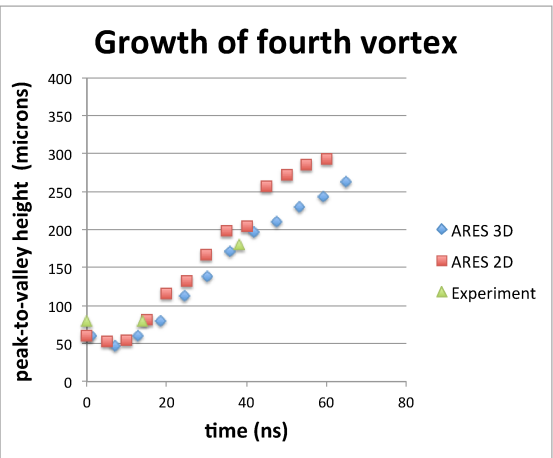

(c)

FIG. 4: Peak-to-valley height vs. time for the three rollups in the experimental field-of-view. The simulations shown here were done in ARES and correspond to the second, third, and fourth crests from the laser drive-side boundary (see Fig. 1). 2D simulation[1] indicated the dynamics of the first crest was highly susceptible to driver-side boundary effects so the field-of-view was focused more downstream, about $1 / 4$ of the way down the shock tube. The experimental data points were inferred from radiographs taken at $25 \mathrm{~ns}, 45 \mathrm{~ns}$, and $75 \mathrm{~ns}$ after the laser pulse. The $75 \mathrm{~ns}$ frame is shown in Fig. 3a while the other frames may be seen in Refs. 2, 3. In each of these plots, the time axis corresponds to the time elapsed after the shock passed the crest. The experimental passage time was inferred from a blast wave model of the shock motion (see Ref. 2 and the discussion in the text) calibrated to the $25 \mathrm{~ns}$ radiograph, in which the shock was actually observed. In each of these plots, the late-time experimental data point is the one inferred, in this manner, from Fig. $3 \mathrm{a}$.

[1] O. A. Hurricane, High Energy Density Physics 4, 97 (2008).

[2] E. C. Harding, J. F. Hansen, O. A. Hurricane, R. P. Drake, H. F. Robey, C. C. Kuranz, B. A. Remington, M. J. Bono, M. J. Grosskopf, and R. S. Gillespie, Phys. Rev. Lett. 103, 045005 (2009).

[3] O. A. Hurricane, J. F. Hansen, H. F. Robey, B. A. Remington, M. J. Bono, E. C. Harding, R. P. Drake, and C. C. Kuranz, Phys. Plasmas 16, 056305 (2009).

[4] O. A. Hurricane, J. F. Hansen, , E. C. Harding, V. A. Smalyuk, B. A. Remington, G. Langstaff, H.-S. Park, H. F. Robey, C. C. Kuranz, et al., Astrophys. Space Sci. (2010).

[5] O. A. Hurricane, in preparation (2012).

[6] V. A. Smalyuk, in preparation (2012).

[7] P. A. Thompson, Compressible-Fluid Dynamics (McGraw-Hill Inc., New York, 1972).

[8] P. E. Dimotakis (AIAA, 1991), Progress in Astronautics and Aeronautics, p. 265.

[9] R. T. Barton (Jones and Bartlett Publishers, Boston, 1985), Numerical Astrophysics, p. 482.

[10] R. M. Darlington, T. L. McAbee, and G. Rodrigue, Comp. Phys. Comm. 135, 58 (2001).

[11] M. B. Aufderheide, G. Henderson, A. E. S. von Wittenau, D. M. Sloane, and H. E. Martz (IEEE, 2004), vol. 4 of 2004 IEEE Nuclear Science Symposium Conference Record, p. 2579.

[12] As part of our mesh strategy, we allowed mesh nodes outside the shock tube to rapidly adjust in order to prevent fast flows outside the shock tube, such as the ablator blowoff on the laser drive-side boundary, from controlling the time step. As a consequence of this, tiny amounts of material would occasionally advect from the outermost zones of the beryllium wall into the essentially void (actually low density air) region surrounding the shock tube. We have verified that this does not affect the shock or vortex dynamics in the region of interest. However, it is difficult to remove these specious beryllium zones (in which the density is typically $10^{-3} \mathrm{~g} / \mathrm{cc}$ or less) in making the visualizations.

[13] Y. B. Zeldovich and Y. P. Raizer, Physics of Shock Waves and High-Temperature Hydrodynamic Phenomena (Academic Press, Inc., New York, 1967).

[14] A. Rikanati, U. Alon, and D. Shvarts, Phys. Fluids 15 (2003).

[15] U. Alon, J. Hecht, D. Ofer, and D. Shvarts, Phys. Rev. Lett. 74, 534 (1995).

[16] The origin of the time variable which enters the shock velocity power law is the beginning of the experiment while the origin of the time variable here is when the shock hits the crest in question. Therefore, these times are related to one another by an offset. By "late enough times", we mean times late enough that we can ignore this offset to leading order

[17] The potential value of such a study was suggested to us, in a slightly different context, by Y. Elbaz and D. Shvarts. 


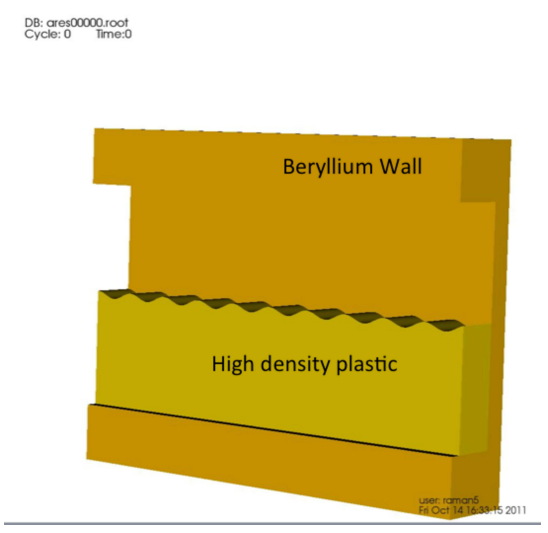

(a) time $=0 \mathrm{~ns}$.

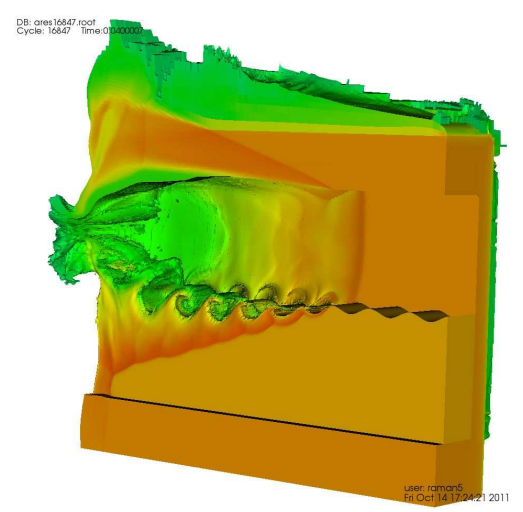

(d) time $=40 \mathrm{~ns}$.

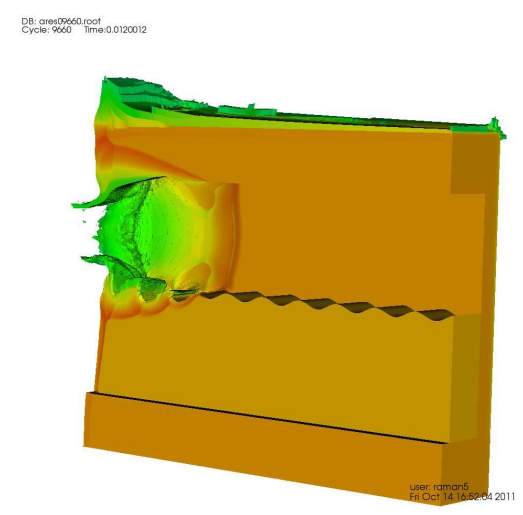

(b) time $=12 \mathrm{~ns}$.

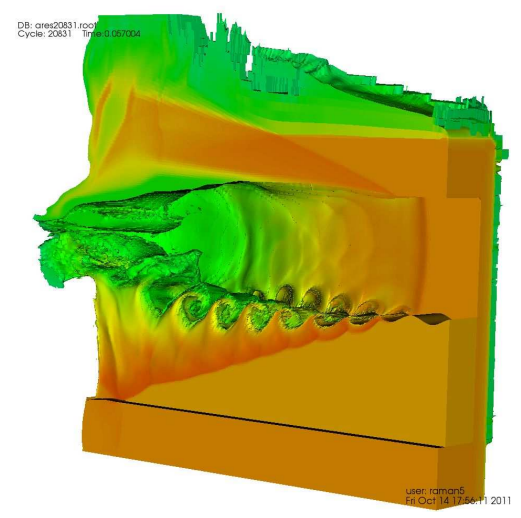

(e) time $=57 \mathrm{~ns}$.

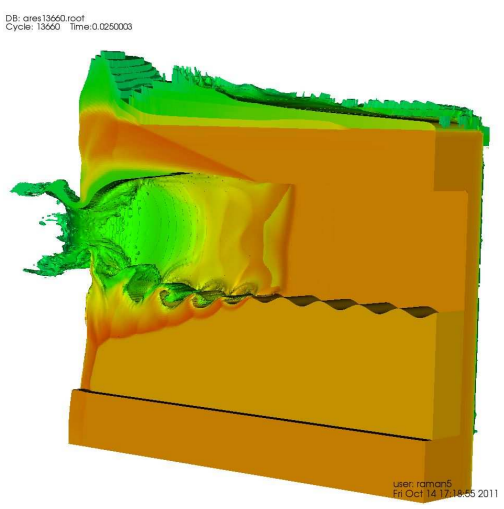

(c) time $=25 \mathrm{~ns}$.

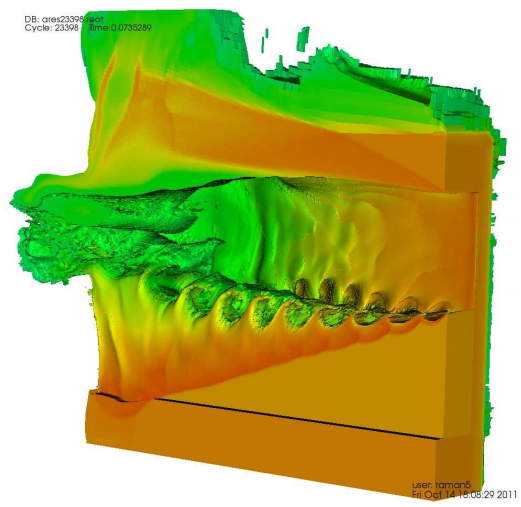

(f) time $=73.5 \mathrm{~ns}$.

FIG. 5: Pseudocolor plot of the logarithm of the density from a 3D ARES simulation. The target has been sliced lengthwise. The beryllium and plastic regions are shown, the foam section, in which the main shock propagates, has been removed in this visualization. Initially, only the plastic part is modulated (a) but the perturbation gets imprinted onto the beryllium wall as the shock moves down the target (frames (b) through (f)). The apparently "acausal" motion on the top of the target is due to the mesh management strategy and should be ignored.[12]

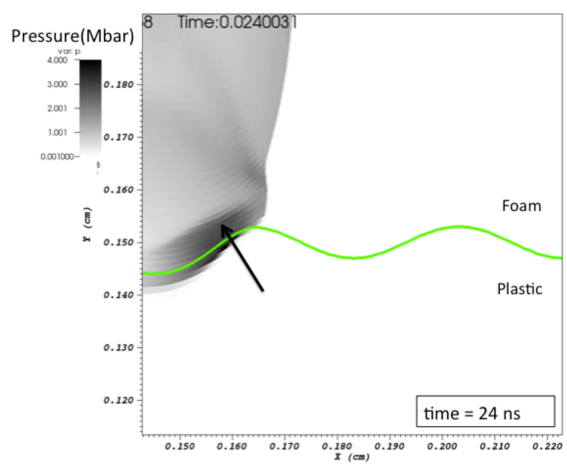

(a)

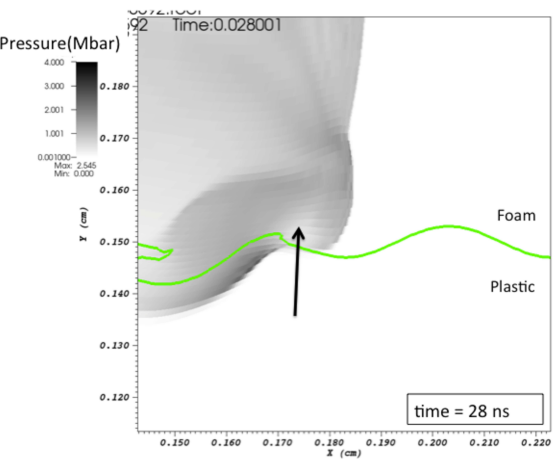

(b)

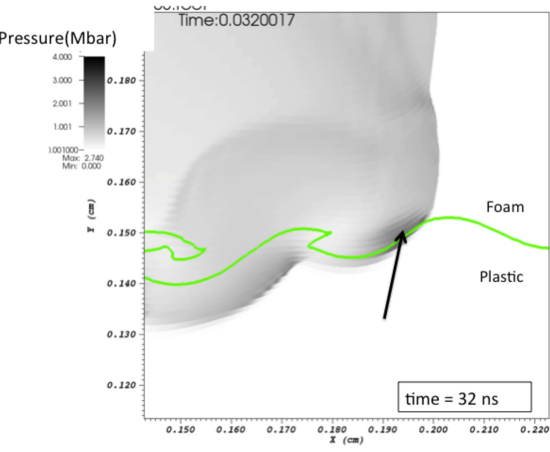

(c)

FIG. 6: Pressure contour plots of the shock going over one of the crests from a 2D ARES simulation. Arrows indicate the local pressure rise (frames (a) and (c)) as the shock climbs up a crest and the corresponding decrease (frame b) as the shock falls down the other side. The local variation in pressure induced by this effect is of comparable magnitude to the freestream pressure itself and strongly affects the beryllium side walls, which lie in the planes above and below the page. 


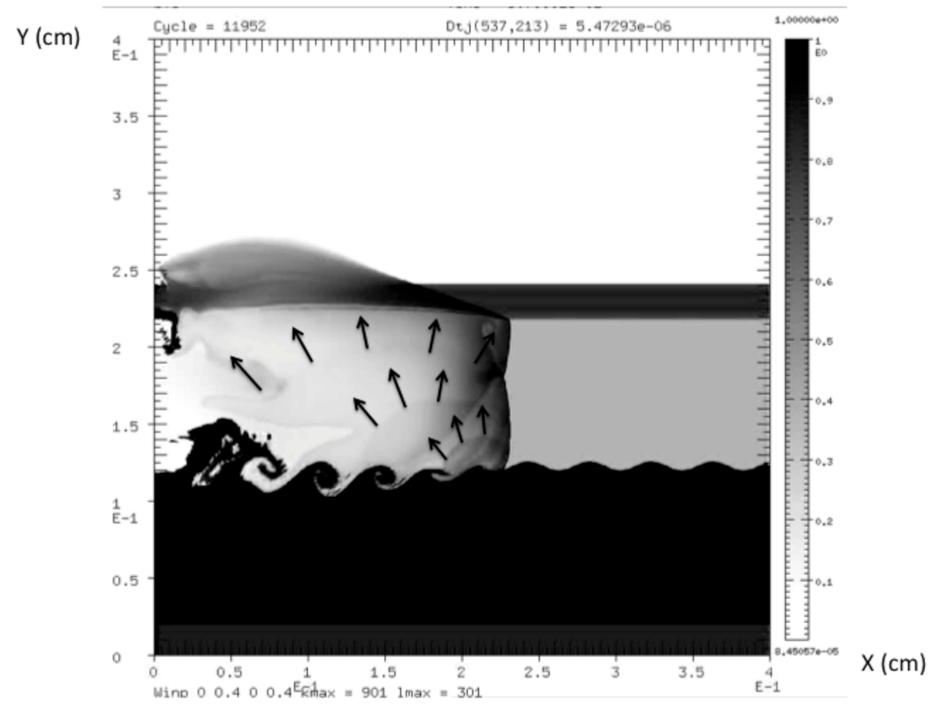

FIG. 7: Synthetic x-ray image from the very high-resolution 2D CALE simulation in Ref. 1. The arrows indicate the outward motion of the spherical reflected shocks emitted from the crests as the main shock passes over. It is difficult to see the outermost wave, which originated from the second crest from the left. The middle wave originated from the third crest and the lower wave from the fourth. These waves are also transmitted into the beryllium side walls lying above and below the page. 

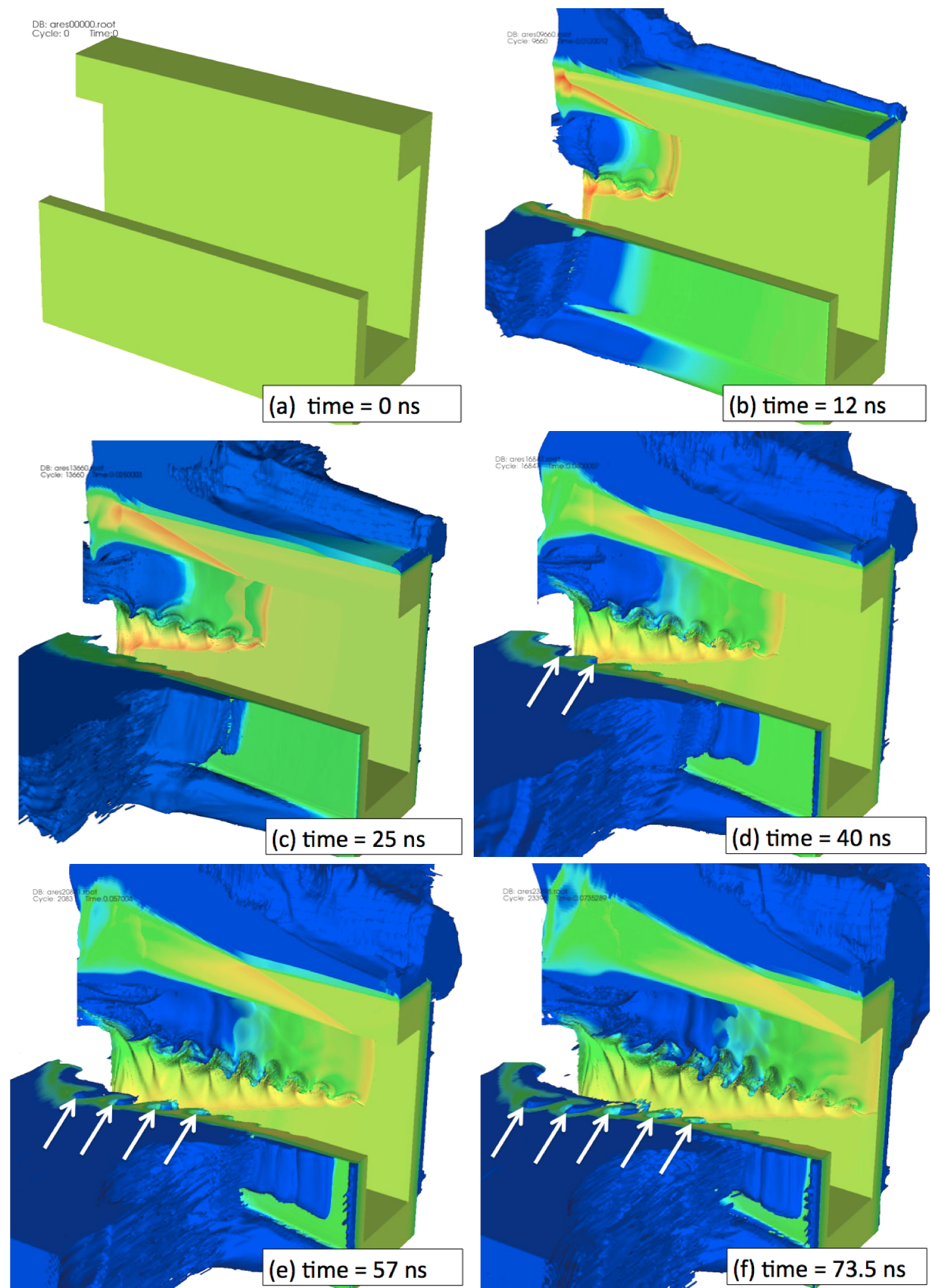

FIG. 8: Log density pseudocolor plot, from our 3D ARES simulation. All material regions inside the beryllium shock tube have been removed in this visualization. The beryllium front wall has been cut to the height of the foam-plastic interface. Pressure modulations are imprinted onto the Be wall at early times (frames (b) and (c)). "Bubbles" in the Be wall are then seen to grow up at later times (frames (d), (e), and (f)). The arrows indicate bubbles which have reached an appreciable size. The apparently "acausal" motions on the top and sides of the target are due to the mesh management strategy and should be ignored.[12] 


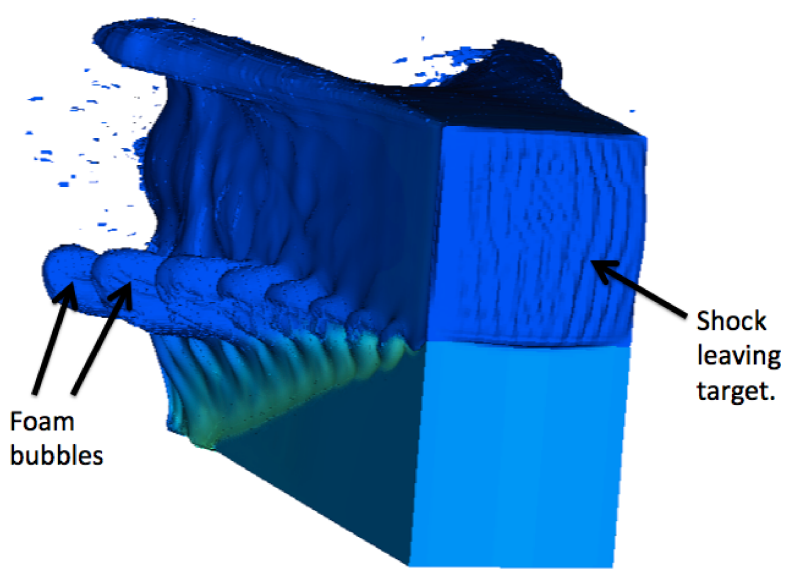

FIG. 9: Density pseudocolor plot from a 3D ARES simulation at late-time (73.5 ns), showing just the foam (top) and plastic (bottom) parts. The sideways growth of the foam bubbles is evident.

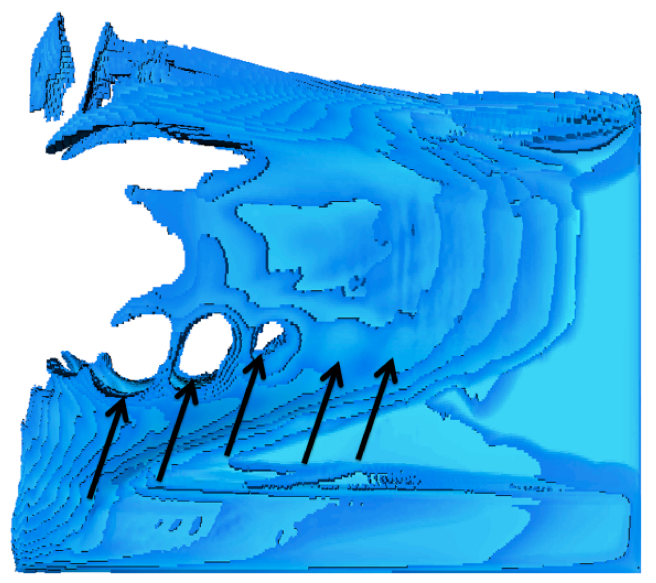

FIG. 10: Threshold density plot of the Be part from a 3D ARES simulation at late-time (73.5 $\mathrm{ns})$. In this visualization, the Be wall is seen from the side (other materials have been removed) and only zones with density greater than $1 \mathrm{~g} / \mathrm{cc}$ are shown. Locations where the foam bubbles have blown through appear as "holes" in the shock tube. The arrows indicate these locations, as well as locations where blowout is imminent. 


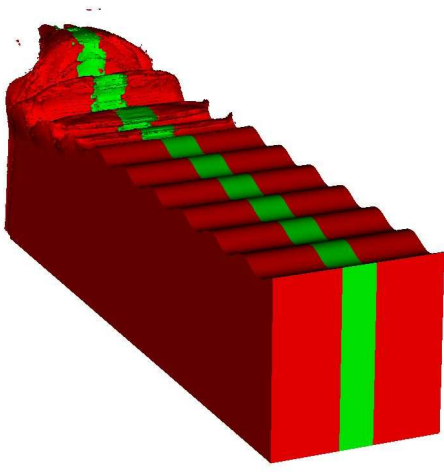

(a) time $=15 \mathrm{~ns}$

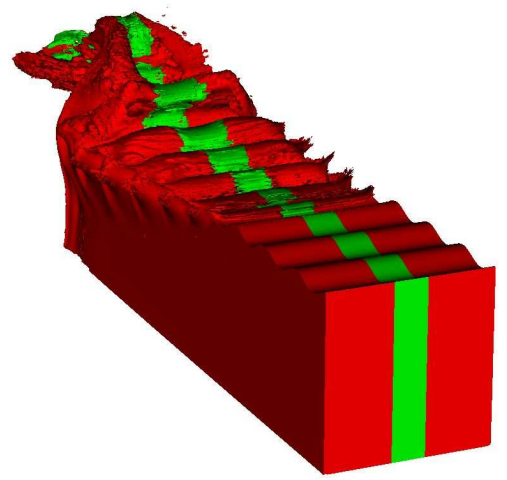

(b) time $=40 \mathrm{~ns}$

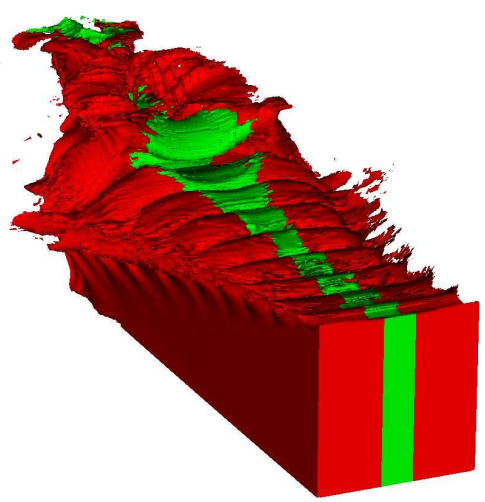

(c) time $=73.5 \mathrm{~ns}$

FIG. 11: Filled material plots of the plastic region from a 3D ARES simulation. In this type of plot, all zones containing the same material are given the same color. The opaque tracer strip along the center line is colored differently. A depression formed in the tracer layer is clearly visible at late time. 


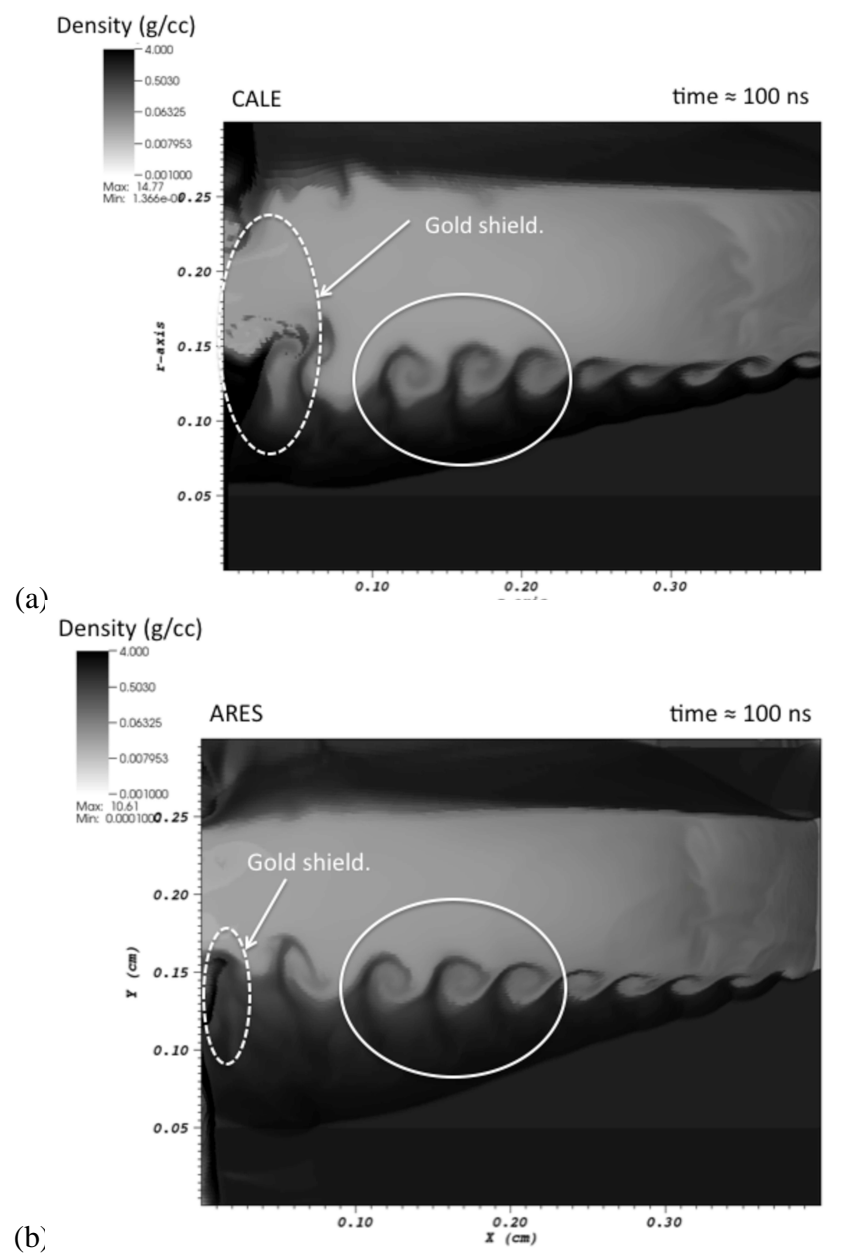

FIG. 12: This figure compares density contour plots, shown on a logarithmic color scale for visualization, from 2D (a) CALE and (b) ARES simulations at very late times. The experimental field-of-view is circled in both plots. As discussed in the text, these simulations used different models for the laser energy deposition and different ALE strategies during the ablation phase, but were otherwise run under the same conditions. While the two simulations are similar, in (a) the flow has drawn the gold shield into the channel more than in (b). This could be related to the rollups in (a) being more prolate, in terms of their height to width, than in (b). 


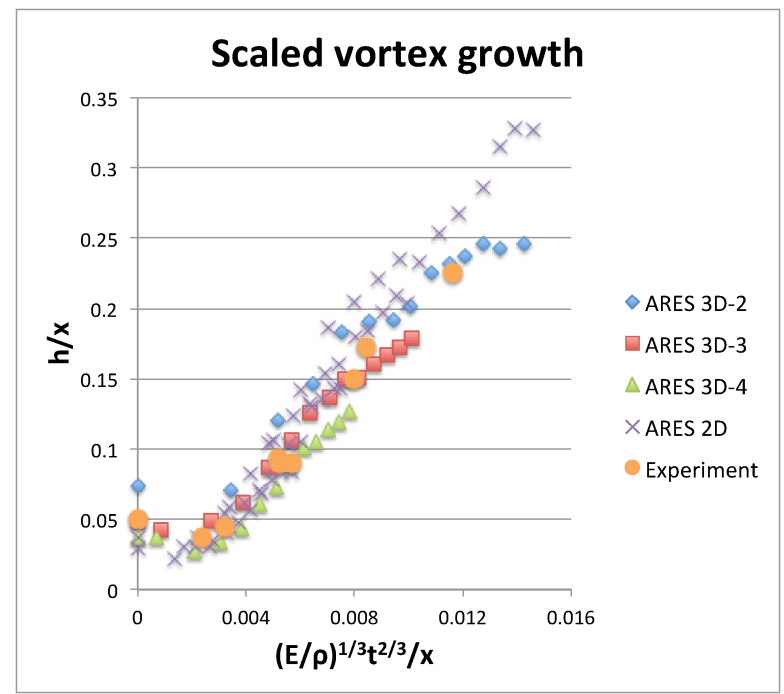

FIG. 13: Vortex growth plotted in non-dimensional variables, as discussed in the text. This figure compares the growths of the three rollups in the experimental field-of-view with values obtained from 2D and 3D ARES simulations. Because the 3D data do not collapse on the same curve like the 2D data, we have plotted the 3D simulation results for the three rollups, labeled 2,3 , and 4 , with different symbols. 